\title{
A Defensive Kicking Behavior in Response to Mechanical Stimuli Mediated by Drosophila Wing Margin Bristles
}

\author{
Jiefu Li, ${ }^{\star}$ Wei Zhang, ${ }^{\star}$ Zhenhao Guo, Sophia Wu, ㄴily Yeh Jan, and $\odot$ Yuh-Nung Jan \\ Departments of Physiology, Biochemistry, and Biophysics, Howard Hughes Medical Institute, University of California, San Francisco, California 94158
}

Mechanosensation, one of the fastest sensory modalities, mediates diverse behaviors including those pertinent for survival. It is important to understand how mechanical stimuli trigger defensive behaviors. Here, we report that Drosophila melanogaster adult flies exhibit a kicking response against invading parasitic mites over their wing margin with ultrafast speed and high spatial precision. Mechanical stimuli that mimic the mites' movement evoke a similar kicking behavior. Further, we identified a TRPV channel, Nanchung, and a specific Nanchung-expressing neuron under each recurved bristle that forms an array along the wing margin as being essential sensory components for this behavior. Our electrophysiological recordings demonstrated that the mechanosensitivity of recurved bristles requires Nanchung and Nanchung-expressing neurons. Together, our results reveal a novel neural mechanism for innate defensive behavior through mechanosensation.

Key words: defensive behavior; Drosophila; touch; trp channel

Significance Statement

We discovered a previously unknown function for recurved bristles on the Drosophila melanogaster wing. We found that when a mite (a parasitic pest for Drosophila) touches the wing margin, the fly initiates a swift and accurate kick to remove the mite. The fly head is dispensable for this behavior. Furthermore, we found that a TRPV channel, Nanchung, and a specific Nanchungexpressing neuron under each recurved bristle are essential for its mechanosensitivity and the kicking behavior. In addition, touching different regions of the wing margin elicits kicking directed precisely at the stimulated region. Our experiments suggest that recurved bristles allow the fly to sense the presence of objects by touch to initiate a defensive behavior (perhaps analogous to touch-evoked scratching; Akiyama et al., 2012).

\section{Introduction}

Animals use their diverse sensory systems to detect the presence of danger. For example, fruit flies use their visual system to detect the approach of predators (Fotowat et al., 2009; Muijres et al., 2014) and their olfactory system to sense chemical signals released from predators, pathogenic microbes, and their startled kin (Suh et al., 2004; Jones et al., 2007; Benton, 2008; Gaudry et al., 2012; Prieto-Godino and Benton, 2013). As one of the fastest

\footnotetext{
Received April 29, 2016; revised Aug. 18, 2016; accepted Sept. 8, 2016.

Author contributions: J.L., W.Z., L.Y.J., and Y.-N.J. designed research; J.L., W.Z., Z.G., and S.W. performed research; J.L., W.Z., and Z.G. analyzed data; J.L., W.Z., L.Y.J., and Y.-N.J. wrote the paper.

This work was supported by BRAINseed (Y.N.J.) and the National Institutes of Health (Grant R37NS040929 to Y.-N.J..). L.Y.J. and Y.-N.J. are investigators of the Howard Hughes Medical Institute. We thank C. Kim and K. Scott for fly lines; S. Younger, T. Cheng, and S. Barbel for technical support; X. Gao, M. Klassen, Y. Song, and W. Luo for comments; and members of the Jan laboratory for discussion.

The authors declare no competing financial interests.

*J.L. and W.Z. contributed equally to this work.

Correspondence should be addressed to Yuh-Nung Jan, Departments of Physiology, Biochemistry, and Biophysics, Howard Hughes Medical Institute, University of California, 1550 4th St. Room 484E, Rock Hall, San Francisco, CA 94158. E-mail: yuhnung.jan@ucsf.edu.

DOI:10.1523/JNEUROSCI.1416-16.2016

Copyright $\odot 2016$ the authors $\quad 0270-6474 / 16 / 3611275-08 \$ 15.00 / 0$
}

sensory modalities, mechanosensation, including hearing and touch sensation, ensures their rapid response to acoustic and tactile stimuli (Effertz et al., 2011; Kim et al., 2012; Yan et al., 2013; Zhang et al., 2013). One example is the ultrafast kicking behaviors of locusts and grasshoppers (Burrows, 1995; Hedwig and Burrows, 1996; Jellema and Heitler, 1999; Sasaki and Burrows, 2003). A locust responds to contact from adversaries in milliseconds by extending the tibiae of its hind legs in a kick with peak angular velocities of $80^{\circ} \mathrm{ms}^{-1}$. The underlying molecular mechanisms and neuronal circuitry that mediate somatosensation to this type of tactile stimulation are an intriguing open question. Although previous studies have reported the neural basis of gentle touch and mechanical nociception of Drosophila melanogaster larvae (Kim et al., 2012; Yan et al., 2013), adult flies exhibit more complex behaviors in response to mechanical stimuli (Vandervorst and Ghysen, 1980; Caldwell and Eberl, 2002; Albert and Göpfert, 2015; Ramdya et al., 2015) and the neural circuits governing these behaviors are not yet well characterized.

Drosophila wings are the flight apparatus of adult flies. They also serve important functions in courtship and aggression (Chen et al., 2002; Yamamoto and Koganezawa, 2013; Yuan et al., 2014). 
These wings cover $>60 \%$ of the dorsal body surface and define the border of a fly's body. Many bristles line the wing margin (Hartenstein and Posakony, 1989) and some are morphologically similar to sensory bristles on other parts of the body. Both mechanical and gustatory sensors may be present in the wing margin (Hartenstein and Posakony, 1989; Valmalette et al., 2015), although the molecular and cellular composition of these sensors remains to be defined.

Here, we report that both invasion of parasitic mites and mechanical touch stimulation on the wing margin trigger an ultrafast kicking behavior that requires a TRPV channel, Nanchung, and an array of Nanchung-expressing neurons along the wing margin. Further, we found that recurved bristles, each innervated by a single Nanchung-expressing neuron, are sensitive to mechanical displacements. The recurved bristle-mediated kicking behavior also exhibits spatial precision. Our results have thus identified a novel function of wing margin bristles and part of the underlying molecular and cellular mechanisms of mechanosensation.

\section{Materials and Methods}

Fly stock. Fruit flies were raised in a $25^{\circ} \mathrm{C}$ incubator (Darwin Chamber Company) with a $12 \mathrm{~h} / 12 \mathrm{~h}$ light cycle and humidity control. The UASNanchung line was provided by Dr. C. Kim (Chonnam National University). The nan ${ }^{36 a}$ and $i a v^{1}$ lines were from Bloomington Stock Center. The NOMPC RNAi line (v105579) was from the Vienna Drosophila Resource Center. The NOMPC RNAi line (B31512) and Deficiency line (3788) were from Bloomington Stock Center. piezo ${ }^{\mathrm{KO}}$ was a gift from Dr. A. Patapoutian at the Scripps Research Institute. $P p k 1$ and $t m c^{\mathrm{KO}}$ were generated in the laboratory as described previously (Gorczyca et al., 2014; Guo et al., 2016). nompC $C^{\text {f00914 }}$ was from Dr. M. Göpfert at University of Gottingen. The GMR lines were generated by the Flylight project (Jenett et al., 2012) and obtained from Bloomington Stock Center.

Behavior assay. Adult flies of either sex (5-7 d after eclosion) were collected. Tethered flies were prepared as described previously (Seelig et al., 2010) with minor modifications. Briefly, the fly was anesthetized on ice and held dorsal side up. An insect pin was attached to the fly notum with low-melting-point wax (Sigma-Aldrich) The head and thorax were glued together to reduce movement. The fly was held in position (either dorsal or lateral side up) by inserting the free end of the insect pin to clay. The fly was then allowed to hold a piece of Kimwipe and rest for $2 \mathrm{~h}$ in a wet chamber before experiments. The behavioral responses to mites or probes were recorded with a high-speed camera (Pointgrey) and the videos were saved for offline analysis.

For studies of headless flies, flies were anesthetized on ice and decapitated with spring scissors (Fine Science Tools). Decapitated flies were kept on a wet tissue pad in a Petri dish for recovery. After $10 \mathrm{~min}$, those flies that were able to stand up and walk were picked up for behavioral test.

In the test with a probe, an eyelash was used to gently touch part of the wing margin so as to mimic the movement of mites. The probe that was perpendicular to the wing blade was moved along the wing margin toward the distal end to provide mechanical stimulation of recurved bristles by causing a small displacement. The probe touched these bristles for $1-2 \mathrm{~s}$ without causing any displacement of the fly. For quantitative analysis, each fly was stimulated 10 times with the stimulation repeated every $10 \mathrm{~s}$ and the number of kicking responses was recorded.

In the test for spatial precision, each fly was stimulated 10 times at the anterior region of the wing margin and 10 times at the posterior region of the wing margin, defined by the L2 vein, and the number of mid-leg and hind leg movements, respectively, was recorded.

Mite invasion and containment. Parasitic mites were cocultured with flies in vials in plastic trays with water on their bottom. Each vial was flipped to a new vial that contained fly food and $\sim 10$ adult flies every 2 weeks. Mites crawling on the wall of the vials were picked up for experimental use. The whole tray was placed on a piece of antimite paper (Carolina Biological).

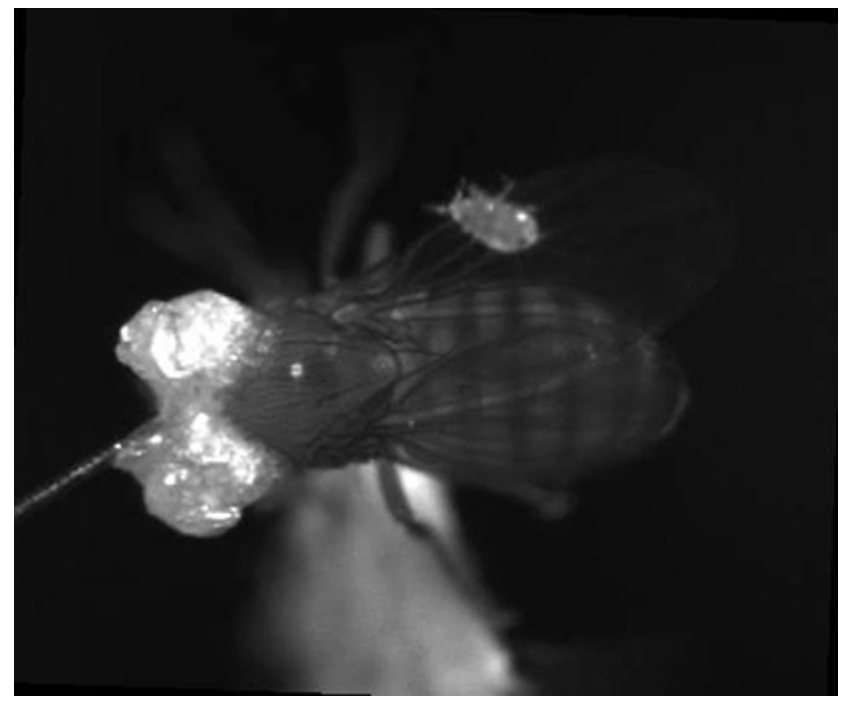

Movie 1. Moving parasitic mite on the wing margin evoked an ultrafast kicking behavior in a tethered fruit fly. Playback speed: slowed down to $1 / 100 \times$.

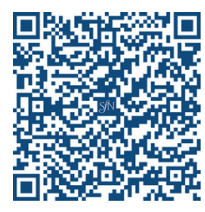

In the behavioral test with parasitic mites, a mite was picked up with tweezers and presented to the fly wing surface with a brush tip. The mite was allowed to crawl freely on the fly body before reaching the wing margin. The behaviors were recorded with a high-speed camera (Pointgrey) and the videos were saved for offline analysis.

Analysis of leg kinematics. Videos were converted to image series with ImageJ. Leg tarsus trajectories were recorded with manual tracking via the ImageJ Manual-Track plugin. Leg kinetics as viewed from the top was characterized. The trajectory angles (intersection angle formed by the lines between the turning point and two most adjacent tarsus positions) were measured with the ImageJ angle tool. The average velocity, from action initiation to kicking on the mite, was calculated with the ManualTrack plugin. The velocity was normalized with the actual body length of the animal.

Confocal microscopy. For imaging, the severed wings and legs of adult flies were picked up with a pair of fine forceps and mounted on a slide with PBS. Images were taken with a $20 \times$ objective in the differential interference contrast channel, as well as in the green and red channels, to visualize the morphology of the bristles.

The ventral nerve cord (VNC) was dissected out of an adult fly, placed in PBS solution, and mounted with its dorsal side up on a slide for imaging. An LSM 510 microscope (Zeiss) with $40 \times$ oil-immersion lens was used for confocal imaging.

Electrophysiological recording. Adult flies (5-7 d after eclosion) were collected for recording from the tips of recurved bristles on the wing margin. Flies were immobilized on coverslips with low melting point wax (Sigma-Aldrich). The abdomen was cut open with a spring scissor and immersed in the saline for recording. Glass electrodes for recording were pulled with a P-97 puller (Sutter Instruments) from precleaned borosilicate glass and filled with saline solution $(128 \mathrm{~mm} \mathrm{NaCl}, 2 \mathrm{~mm} \mathrm{KCl}$, and 5 mм HEPES, adjusted to $\mathrm{pH}$ 7.2). The glass electrode was placed onto the tip of a recurved bristle. The same electrode was used to move the tip of the bristle from the original tension-free position toward the distal end to displace the tip by $40 \mu \mathrm{m}$. Clampex version 10.3 software (Molecular Devices) was used for data acquisition and processing. Clampfit was used to detect neuronal firing automatically based on threshold searching. Briefly, thresholds (estimated manually based on the spike amplitudes emerging after stimulation) were set below the baseline and all negativegoing spikes crossing the threshold were counted.

Graph plotting and statistical analysis. GraphPad Prism 6 was used for graph plotting and statistical analysis. Behavioral scores were grouped for 
A

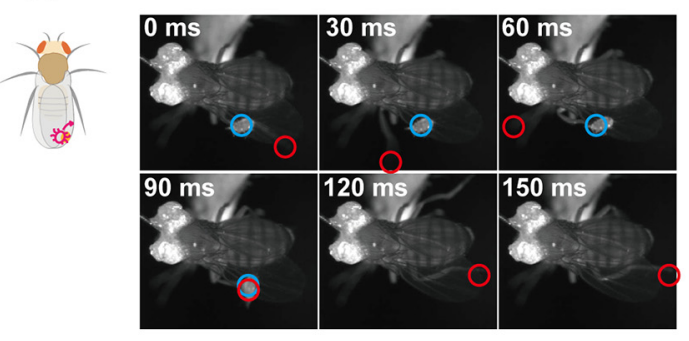

C

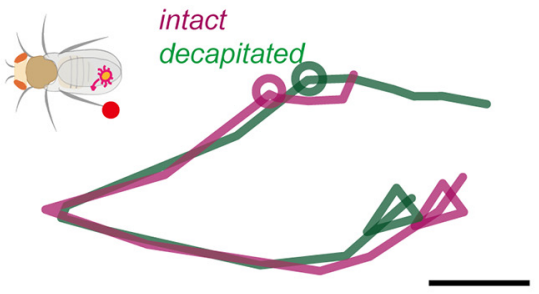

$\mathbf{E}$

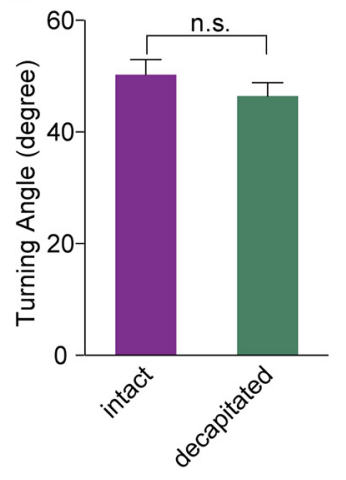

$\mathbf{F}$

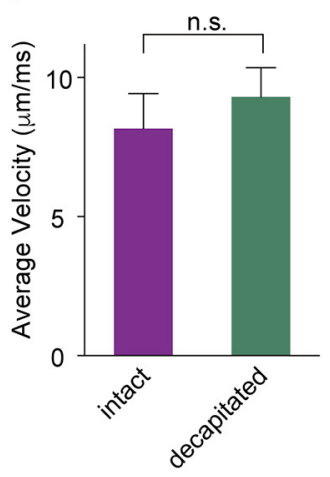

B

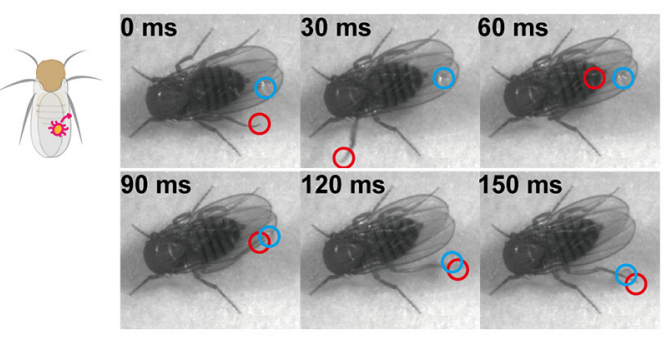

D

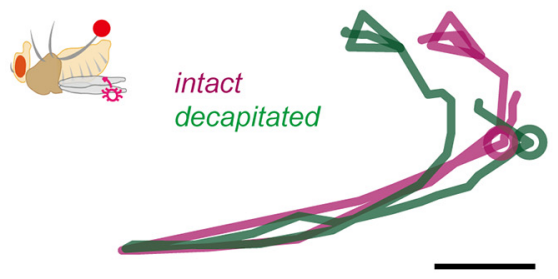

G

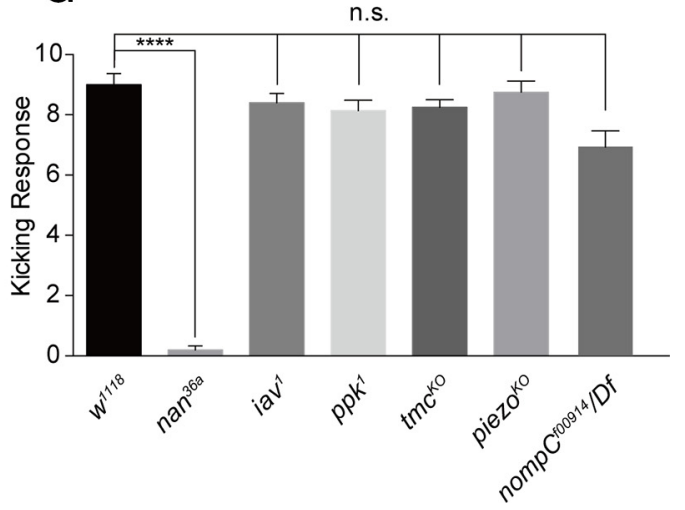

Figure 1. Fruit flies respond to mechanical stimuli on their wing margin. $A$, A tethered fruit fly kicked a mite off its wing margin. Blue circle, mite; red circle, distal segment of the left hind leg. $\boldsymbol{B}$, A decapitated fruit fly kicked a mite off its wing margin. Blue circle, mite; red circle, distal segment of the left hind leg. $C$, Trajectories from the top view of the hind-leg tarsi during kicking. Turning points were aligned together for comparison. Circles indicate the mite position. Arrowheads point to the initiation direction of leg movement. Scale bar, $0.5 \mathrm{~mm}$. $\boldsymbol{D}$, Trajectories from the lateral view of the hind-leg tarsi during kicking. $\boldsymbol{E}, \boldsymbol{F}$, Angles at turning points of the trajectories and average velocity during kicking ( $n=7$; two-tailed, unpaired $t$ test; n.S., not significant). Error bars indicate SEM. G, Probe-induced mechanical stimuli on wing margin evoked the kicking behavior. The nanchung mutant ( nan $^{36 \mathrm{a}}$ ) was defective in the response, whereas the other mutants iav ${ }^{1}$, ppk ${ }^{1}$, $\mathrm{tmc}^{\mathrm{K} 0}$, piezo ${ }^{\mathrm{KO}}$, and nomp $\mathrm{C}^{\mathrm{f} 00914}$ and were relatively normal ( $n=9-10$; two-tailed, unpaired $t$ test was used for comparison; n.S., not significant; $\left.{ }^{* * * *} p<0.0001\right)$. Error bars indicate SEM. Fly genotypes:

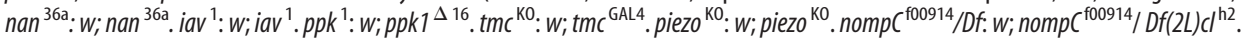

statistical analysis to assess the difference between genotypes. ANOVA followed by Tukey's test was used for comparison between multiple groups. Two-tailed, unpaired $t$ test was used for two-group comparisons. All error bars in the figures indicate SEM.

\section{Results}

Parasitic mites present threats to many insect species (Houck et al., 1991; Martin et al., 2012). Here, we report that an adult fly is able to sense mites crawling on its wings. As shown in Movie 1, a fly tethered to an insect pin held a small piece of paper by its legs, so losing grip of the paper would be an indication of its attempt to jump. When a mite touched its anterior wing margin, it kicked the mite off with a stereotyped behavioral sequence as follows. First, the fly sensed the presence of the moving mite immediately after the mite reached its wing margin (Fig. 1A). Then, the fly then initiated a series of actions within tens of milliseconds by pulling back the body, swinging the hind leg on the ipsilateral side and kicking the mite off the wing (Fig. 1A, Movie 1). The entire behavioral sequence was completed within 200 millisecondsfast enough to kick off a moving mite. The tethered flies exhibited no obvious jump (dropping the paper held by the legs), grooming, or flight (flipping the wing).

To better characterize this kicking response, as well as to minimize potential disturbance arising from other sensory inputs to the head, we placed a freely moving parasitic mite on the wing surface of a decapitated fly, which can stay alive and remain standing and responsive for hours without walking or flying away, and recorded the behavioral sequence. As shown in Figure $1 B$, Movie 2, and Movie 3, the decapitated flies were able to kick the mites as precisely and quickly as the intact flies did. Decapitated flies and intact flies displayed similar trajectory patterns (Fig. 1C,D), similar angles at the anterior turning points (Fig. 1E), and comparable average velocities of the hind-leg tarsus (measured from the initiation of action to the kicking of the mite, based on viewing from above the tarsus trajectory; Fig. $1 F)$. Furthermore, the leg kinematics depended on the position of the mite because both intact flies and decapitated flies were able to adjust their leg posture to target the mites precisely (Fig. 1C,D). 


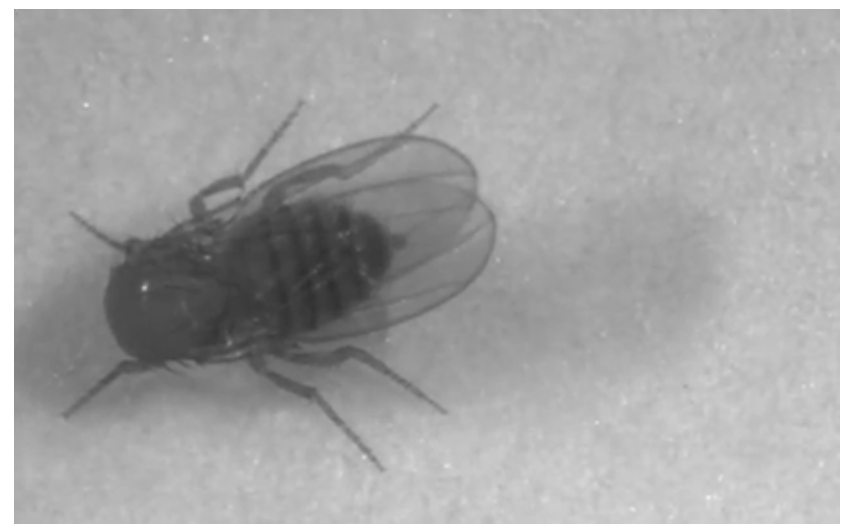

Movie 2. Moving parasitic mite on the wing margin evoked an ultrafast kicking behavior in a decapitated fruit fly. Playback speed: original $1 \times$.
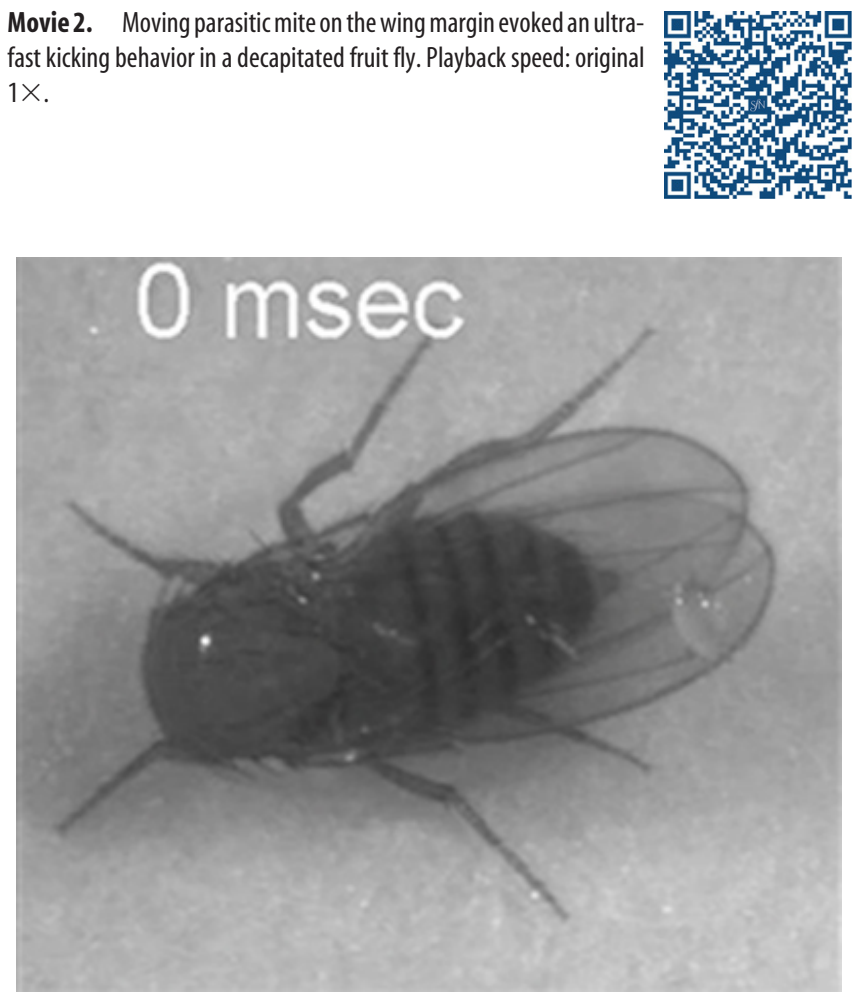

Movie 3. Moving parasitic mite on the wing margin evoked an ultrafast kicking behavior in a decapitated fruit fly. Playback speed: slowed down to $1 / 100 \times$.

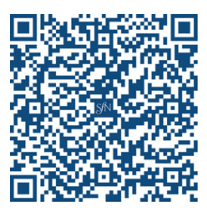

The wing margin may be equipped with both mechanical and gustatory sensors (Hartenstein and Posakony, 1989; Valmalette et al., 2015) that could potentially be activated by a mite. To search for the mechanosensory components of this kicking behavior, we used a probe to generate mechanical stimulation on the wing margin without activating taste neurons. We found that touching the wing margin with a probe evoked the same kicking behavior as that elicited by a mite (Movie 4), suggesting that this defensive behavior is mediated by mechanosensory structures on the wing margin. Moreover, the fly initiated the response as soon as the mite touched the wing margin bristles with its anterior body part (its forelegs or palp), suggesting that wing margin bristles sense subtle displacement rather than the bodyweight of the mite (Movies 1, 2, and 3).

To elucidate the molecular mechanism of this response, we applied the probe assay to mutants of candidate mechanotrans-

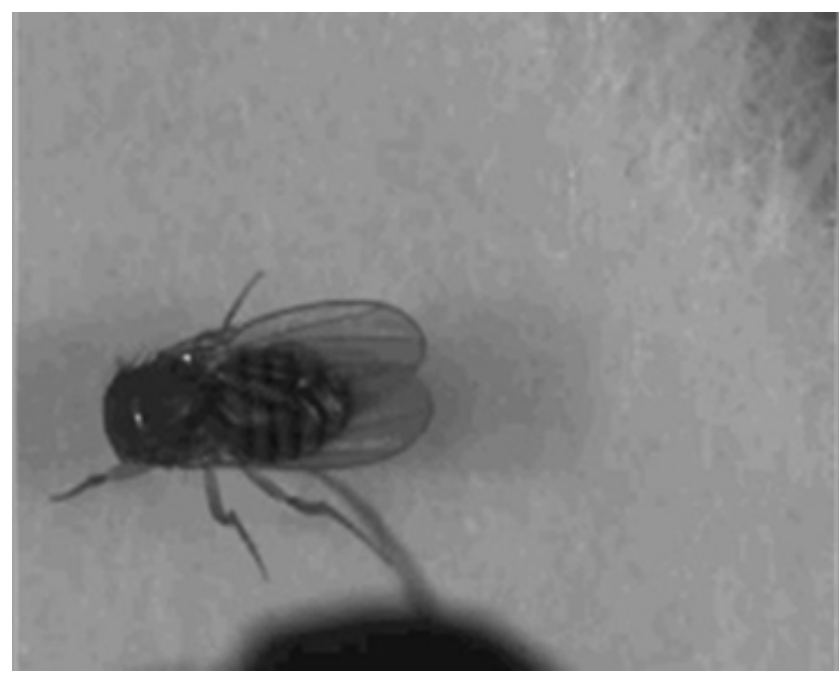

Movie 4. A probe mimicking mechanical stimuli on the wing margin evoked the same kicking behavior. Playback speed: original 1X.
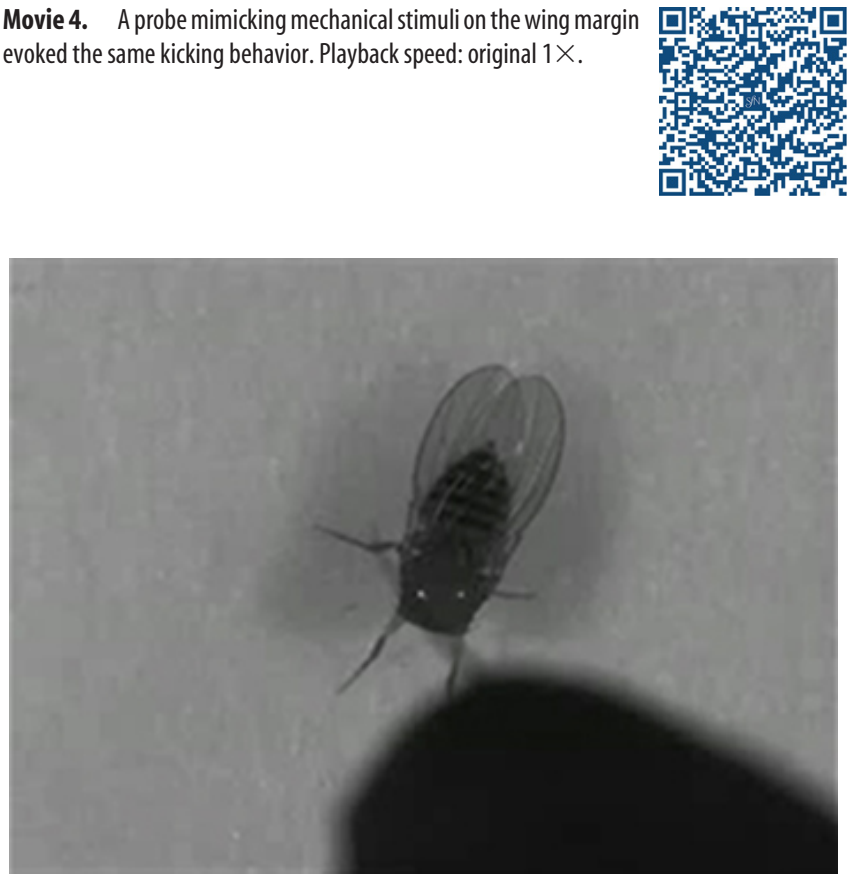

Movie 5. Nanchung mutant $\left(\right.$ nan $\left.^{36 a}\right)$ flies do not respond to the mechanical stimuli on the wing margin. Playback speed: original $1 \times$.

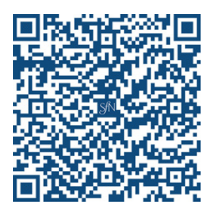

duction channel genes. Compared with wild-type flies, mutant flies lacking the TRPV channel Nanchung (nan ${ }^{36 a}$ ) displayed greatly reduced kicking responses to the probe stimulation (Fig. $1 G$, Movie 5). In contrast, mutants of several other mechanosensitive channel genes, including piezo (Kim et al., 2012), Ppk1 (Gorczyca et al., 2014; Guo et al., 2014), nompC (Walker et al., 2000; Yan et al., 2013; Ramdya et al., 2015) and tmc (Guo et al., 2016), exhibited normal responses. Intriguingly, flies lacking Inactive $\left(i a v^{1}\right)$, a partner of Nan in the mechanotransduction for hearing of the fly (Gong et al., 2004), also showed normal kicking responses (Fig. 1G), suggesting an Iav-independent function of Nan in mechanotransduction at the wing margin.

By labeling Nan + neurons with a Nan-Gal4 line, we observed that there is one Nan + neuron (labeled with Red-Stringer that 


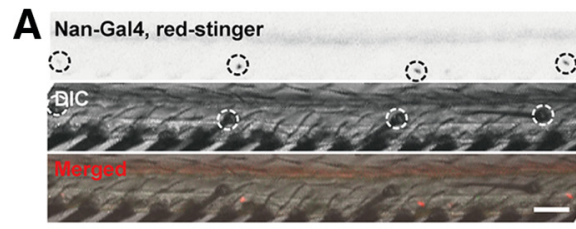

D

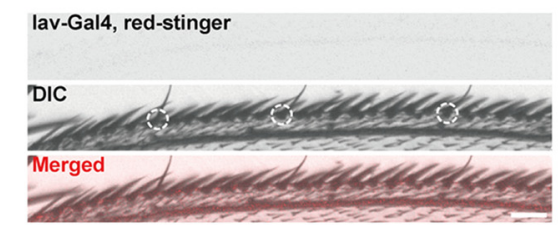

B

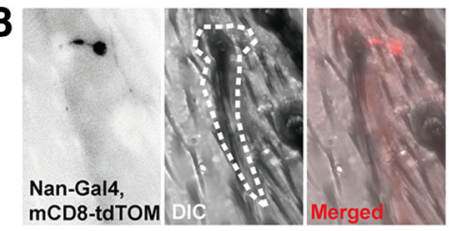

E

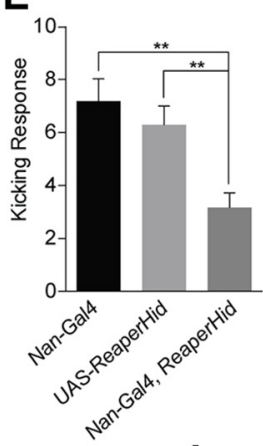

$\mathbf{F}$

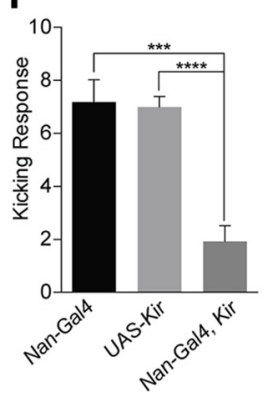

C

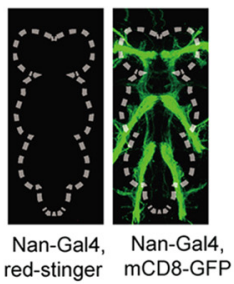

G

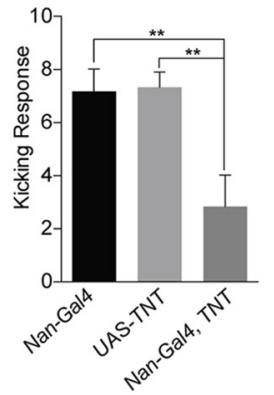

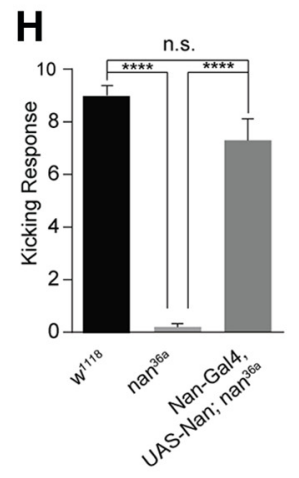

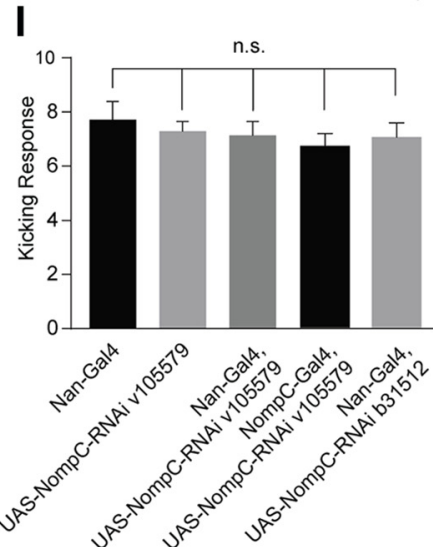

J K

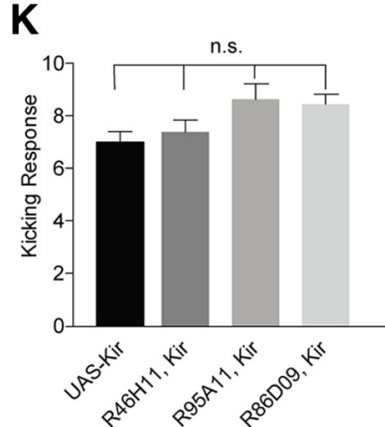

Figure 2. Neuronal basis of the defensive behavior triggered by wing margin stimulation. $A, B$, Nanchung-specific Gal4 labels an array of neurons that exhibit one-to-one innervation to the recurved bristles along the wing margin. Black circle, nuclei; white circle/dashed line, recurved bristles. Scale bars, $20 \mu \mathrm{m}$. Nan-Gal4, red-stinger: Nan-Gal4/UAS-red-stinger; Nan-Gal4, mCD8-tdTOM: Nan-Gal4/UAS-mCD8tdTomato; DIC, differential interference contrast microscopy. C, Nan-Gal4 shows no labeling of neuronal cell bodies in the adult VNC. Left, Nan-Gal4/UAS-red-stinger. Right, Nan-Gal4/UAS-mCD8-GFP. White dashed lines outline the border of the VNC. D, lav-specific-Gal4 shows no labeling of neurons along the wing margin. White circle, recurved bristles. Scale bars, $20 \mu \mathrm{m}$. lav-Gal4, red-stinger: lav-Gal4/UAS-redstinger. $\boldsymbol{E}-\mathbf{G}$, Ablating or silencing Nan + neurons with Reaper-Hid (E), Kir2.1 ( $\boldsymbol{F})$, and TNT ( $\boldsymbol{G})$ expression causes defective defensive behavior against mechanical stimuli $(n=7-11$; two-tailed, unpaired $t$ test was used for comparison; ${ }^{* *} p<0.01 .{ }^{* * *} p<0.001$. ${ }^{* * *} p<0.0001$ ). Error bars indicate SEM. Nan-Gal4, ReaperHid:Nan-Gal4/UAS-ReaperHid.Nan-Gal4, Kir:Nan-Gal4/UAS-Kir2.1.Nan-Gal4, TNT:Nan-Gal4/ UAS-TNT. H, Expression of NAN protein with Nan-Gal4 rescued the behavioral phenotype of nanchung mutant ( $n=9$-14; one-way ANOVA followed by Tukey's sest was used for comparison; $n$.S., not significant; ${ }_{* * * *}<0.0001$ ). Error bars indicate SEM. Nan-Gal4, UAS-Nan; nan ${ }^{36 a}$ :Nan-Gal4/UAS-Nanchung; nan $^{36 a} /$ nan $^{36 a}$.I, Knocking down NOMPC with Nan-Gal4 or nompC-Gal4 does not change the kicking response ( $n=7-8$; two-tailed, unpaired $t$ test was used for comparison; n.S., not significant). Error bars indicate SEM. UAS-NompC-RNAiv105579:UAS-NompC-RNAiv105579/+; UAS-Dcr2/+. Nan-Gal4, UAS-NompCRNAiv105579:Nan-Gal4/UAS-NompC-RNAiv105579; UAS-Dcr2/+. Nan-Gal4, UAS-NompC-RNAib31512:Nan-Gal4/UAS-NompC-RNAib31512; UAS-Dcr2/+. nompC-Gal4, UAS-NompC-RNAiv105579:nompCGal4/UAS-NompC-RNAiv105579; UAS-Dcr2/+.J, Leg-CHO Gal4 lines label joints between fore-leg femur and tibia but not wing margin bristles. $K$, Silencing leg CHO neurons with Kir2.1 did notimpair the kicking behavior. R46H11, Kir: R46H11-Gal4/UAS-Kir2.1. R95A11, Kir: R95A11-Gal4/UAS-Kir2.1. R86D09, Kir: R86D09-Gal4/UAS-Kir2.1 ( $n=7$-10; two-tailed, unpaired $t$ test was used for comparison; n.S., not significant). Error bars indicate SEM.

marks the nucleus) under each recurved bristle, with its dendrites (labeled with mCD8-tdTomato that marks the cell membrane) projecting into the bristle cavity (Fig. 2A,B). Whereas Nan+ neurons in the periphery send their axons into the VNC, as evident from labeling by the membrane reporter mCD8-GFP, the Nan-Gal4 line revealed no Nan + cell bodies in the VNC, as evident from labeling of the nuclear reporter Red-Stinger (Fig. 2C), excluding the possibility that the nan mutant phenotype was due to a defect in the central neural circuit. In contrast, there was no detectable expression of Iav at the wing margin, as revealed by Iav-Gal4 labeling, further validating that Nan functions in an Iav-independent manner (Fig. 2D).

To test whether Nan+ neurons in the wings are required for the touch-evoked kicking behavior, we used Nan-Gal4 to drive the expression of Reaper-Hid, which induces apoptosis (McNabb
Table 1. Summary of neuronal numbers in recurved bristles on wing margin

\begin{tabular}{lll}
\hline Gal4 line & Neuronal type & Cell no. in recurved bristles \\
\hline ChaT-Gal4 & Cholinergic & $\sim 4-5$ \\
Elav-Gal4 & Pan-neuronal & $\sim 4-5$ \\
Nan-Gal4 & Nanchung + & 1 \\
lav-Gal4 & Inactive + & 0 \\
nompC-Gal4 & nompC + & 0 \\
Tubulin-Gal4 & Gustatory $^{a}$ & Undefined \\
\hline
\end{tabular}

${ }^{a}$ Valmalette et al., 2015; Raad et al., 2016.

et al., 1997; Goyal et al., 2000), and found that the flies with Nan+ neurons ablated showed impaired defensive behavior in response to touch on the wing margin (Fig. 2E). Further, silencing the $\mathrm{Nan}+$ neurons or interfering with their synaptic transmission via 
A
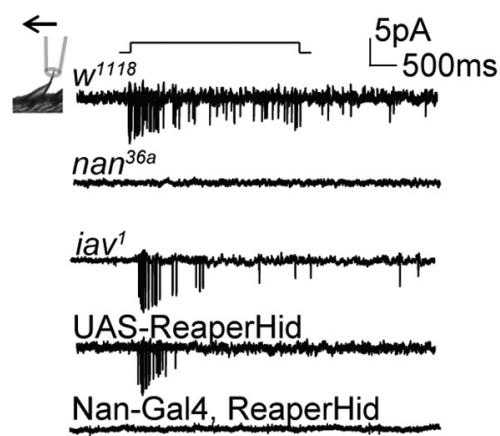

B

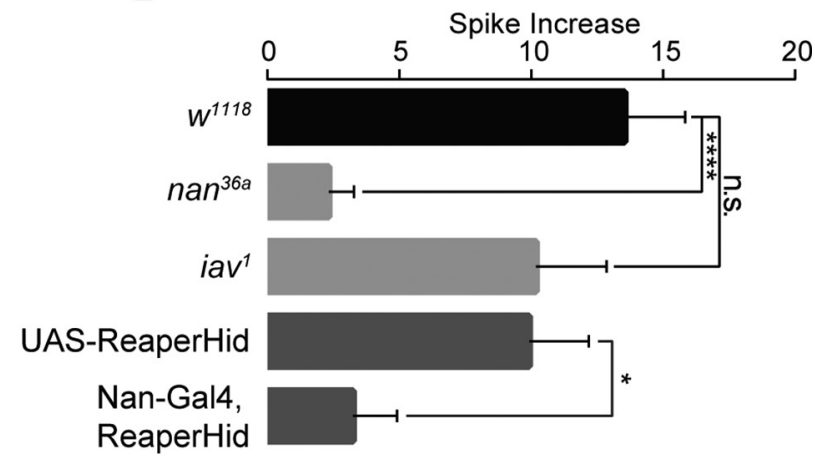

Figure 3. Recurved bristles are mechanosensitive. $\boldsymbol{A}, \boldsymbol{B}$, Mechanical displacement of $40 \mu \mathrm{m}$ induced spike firing in the recurved bristle neurons. nanchung mutant or ablation of Nan + neurons showed reduced firing response, whereas the inactive mutant (iav ${ }^{1}$ ) was normal ( $n=11-15$; two-tailed, unpaired $t$ test was used for comparison; $n$. s., not significant; ${ }^{*} p<0.05$. $\left.{ }^{* * *} p<0.0001\right)$. Error bars indicate SEM. Nan-Gal4, ReaperHid: Nan-Gal4/UAS-ReaperHid.
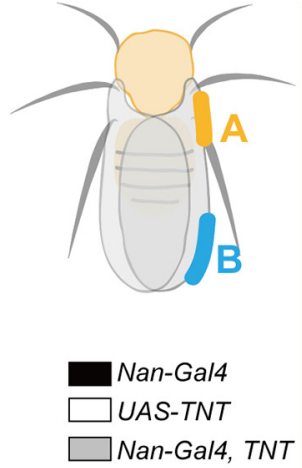

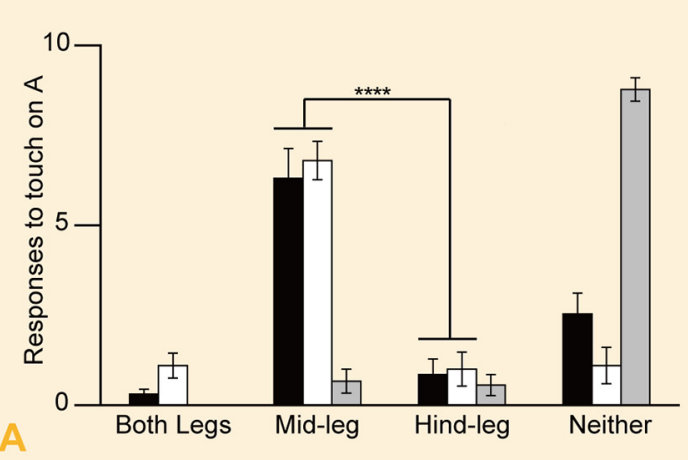

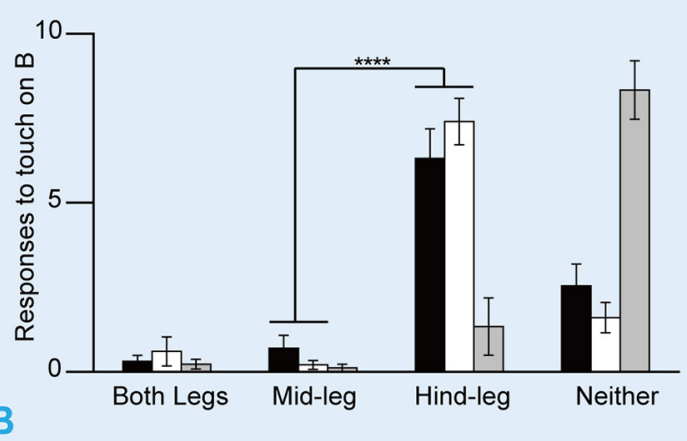

Figure 4. The recurved bristle-mediated defensive behavior exhibits spatial precision. Fruit flies used mid-legs or hind legs, respectively, in responding to mechanical stimuli on the proximal $(\boldsymbol{A})$ or distal $(\boldsymbol{B})$ part of wing margin ( $n=9-13$ ). The Nan-Gal4 and UAS-TNT datasets (both flies exhibited normal behaviors) were grouped for statistical analysis to assess the difference between behavioral patterns (mid-leg or hind leg) when different wing region (A or B) was touched. Two-way ANOVA followed by Tukey's test was used for comparison; ${ }^{* * *} p<0.0001$. Error bars indicate SEM. Nan-Gal4, TNT: Nan-Gal4/UAS-TNT.

the expression of Kir2.1 or TNT, respectively, also reduced this kicking response (Fig. $2 F, G$ ). The remaining responses could reflect incomplete neuronal silencing. Moreover, whereas nanchung mutants $\left(\right.$ nan $\left.^{36 a}\right)$ exhibited no kicking response, expression of the full-length NAN protein driven by Nan-Gal4 in the mutant background fully rescued the behavioral phenotype (Fig. $2 \mathrm{H}$ ), suggesting that Nan functions in the Nan-Gal4 labeled neurons to mediate this kicking behavior. We also tested the involvement of NOMPC, a mechanotransduction channel involved in gentle touch of Drosophila larvae (Yan et al., 2013; Zhang et al., 2015), by using multiple RNA interference (RNAi) lines for RNAi knockdown of NOMPC in the Nan+ neurons, and found that NOMPC channels are not required for this kicking response (Fig. 2I). Knock-down of NOMPC with a nompC-Gal4 also yielded a similar result (Fig. 2I).

Nan was reported to reside in chordotonal organ (CHO) neurons, including those on the legs (Mendes et al., 2013). To test whether leg $\mathrm{CHO}$ neurons are important for the kicking behavior, we silenced them with Gal4 lines that label leg $\mathrm{CHO}$ neurons (Jenett et al., 2012), but not wing margin neurons (Fig. 2J). The flies with silenced leg $\mathrm{CHO}$ neurons showed normal kicking responses (Fig. $2 K$ ). These findings are consistent with the notion that Nan functions primarily in wing margin bristles to mediate the behavior.

Although a single Nan + neuron was found to innervate each recurved bristle, there were a number of cell bodies under each recurved bristle, as revealed by labeling via a pan-neuronal ElavGal4 (Table 1). The cholinergic neuronal marker ChaT-Gal4 revealed a similar pattern. Some of these neurons have been reported to be gustatory neurons (Dunipace et al., 2001; Valmalette et al., 2015). To test whether recurved bristles are indeed mechanosensitive, we performed extracellular recording over this structure (Fig. 3A). When a recurved bristle was bent with a $40 \mu \mathrm{m}$ displacement of its tip, an increase of spike firing was observed (Fig. $3 A$ ), indicating that recurved bristles are mechanosensitive. Next, we recorded the neuronal activity of recurved bristles in the nan-null mutant $\left(\right.$ nan $\left.^{36 a}\right)$ flies and found no response to the displacement of a magnitude that was sufficient to induce spikes in wild-type bristles (Fig. $3 A, B$ ). In contrast, the mechanosensitivity of recurved bristles in the inactive-null mutant $\left(\right.$ iav $\left.^{1}\right)$ was similar to that of wild-type controls (Fig. $3 A, B$ ), lending support to the notion that Nan functions independently of Iav in the mechanotransduction of recurved bristles on the wing margin. Moreover, ablation of Nan + neurons with ReaperHid resulted in defective mechanosensitivity of recurved bristles (Fig. $3 A, B$ ). Together, these results indicate that Nan+ neurons are the mechanosensory neurons that innervate recurved bristles along the wing margin to mediate the kicking response.

In addition to the ultrafast speed of response, the kicking behavior also exhibited high spatial accuracy (Movies 1,2), enabling the fly to get rid of small pests. To characterize the spatial precision of the behavior induced by stimulating the wing margin recurved bristles, we divided the wing margin into two regions: the proximal costal wing (marked as " $A$ " in Fig. 4) and the distal wing margin between the wing veins L2 and L3 (marked as "B" in Fig. 4). We stimulated the two regions separately and scored the behavioral responses and found that stimulating different regions evoked distinct responses. 
Flies mainly used their hind legs to kick the probe when the distal margin was touched, whereas mid-leg extension was the typical response when the costal wing was touched (Fig. 4). Our results indicate that flies are able to distinguish spatial information conveyed by mechanical stimulation of recurved bristles in different parts of the wing margin and respond accordingly.

\section{Discussion}

Together, our experiments showed that adult fruit flies use the mechanosensitive recurved bristles, each innervated by a neuron expressing the TRPV channel Nan, to detect gentle tactile stimulation along the wing margin and evoke a defensive behavior against invading objects. Our study revealed the structural, cellular, and molecular components underlying a newly identified defensive behavior involving somatosensory signaling of adult fruit flies. This temporally ultrafast, spatially precise behavioral response to gentle disturbance caused by mechanical stimuli allows the flies to protect themselves from potentially harmful objects, including parasitic mites.

In addition to recurved bristles, there are two other types of bristles on the wing margin: the stout bristle and the slender bristle (Hartenstein and Posakony, 1989). We have found that the behavioral response to mechanical stimulation on the wing margin requires both $\mathrm{Nan}+$ neuronal activity and Nan channel function, indicating that the recurved bristles that are innervated by Nan + neurons are essential for this defensive behavior. Although other types of bristles may be mechanosensitive, they do not express Nan and thus are unlikely to be involved in the Nandependent kicking behavior. Whereas Nan-Gal4 also labels neurons in the mouth and legs, the behavior observed here was triggered specifically by local stimulation at the wing margin, indicating that the Nan+ neurons on this structure are essential.

\section{Kicking behavior versus grooming behavior}

Fruit flies exhibit stereotypical grooming behavior when their body surface is contaminated with foreign particles such asdust (Corfas and Dudai, 1989; Kays et al., 2014; Seeds et al., 2014). A major feature of the grooming behavior is the repetitive cleaning actions over a relatively large area of the body surface. In contrast, the kicking behavior observed in this study was rarely repetitive. Flies were able to remove the mite or kick the probe in one single move. In addition, the kicking behavior was achieved with a powerful stroke by bringing the leg to a defined spatial point, not all over the wing. These features distinguish the kicking behavior from grooming. Previous studies indicate that flies are able to clean dust on their wings via a grooming behavior, which might involve mechanosensation on the wing (Seeds et al., 2014). Although it is possible that the kicking behavior and the grooming behavior share common sensory pathways, global or persistent stimulation appears more likely to recruit the grooming behavior.

Kicking behaviors involving rapid leg extension have been reported in other insects (Burrows, 1995; Jellema and Heitler, 1999; Sasaki and Burrows, 2003). The behavior observed in our study exhibits, not only fast temporal response, but also high versatility because flies target the mites precisely by training the trajectory of their mid-leg or hind leg, depending on the location of the mite on the wing. In mammals, light touch of hairy skin evokes a type of itch termed mechanical itch, which can elicit discrete hind-limb scratch directed toward the stimulus (Green and Dong, 2016). The fast kicking behaviors and itch-evoked scratching are likely of ethological relevance in defense against adversaries.

\section{Inactive and independent role of Nanchung}

Previous studies have found that Nan and Iav are obligate partners in hearing transduction of larvae and adult flies and these two proteins are mutually dependent in their subcellular localization and function (Kim et al., 2003; Gong et al., 2004; Lehnert et al., 2013; Zhang et al., 2013). Although they always coexist in chordotonal organ neurons, whether Nan has any other functions in other neurons independently of Iav is an open question. Here, we found that, in neurons innervating recurved bristles of the wing margin, Nan functioned in mechanotransduction in the absence of Iav. It is possible that Nan itself is sufficient to fulfill the channel function in these neurons as it does in a heterologous system (Kim et al., 2003). It is also possible that there is a different molecular partner(s) for Nan other than Iav.

\section{Transduction mechanisms of mechanosensory bristles}

Drosophila ciliated sensory neurons (type I sensory neurons) are reminiscent of ciliated mechanosensitive cells in other organisms, including amphid neurons in Caenorhabditis elegans and hair cells in the auditory system of vertebrates, thus providing an amenable system with which to investigate the molecular mechanisms in ciliated mechanosensory neurons (Jarman, 2002). Previous studies have shown that sensory bristles on the fly notum rely on NOMPC for their mechanotransduction (Kernan et al., 1994; Eberl et al., 2000). Here, we report that another TRP channel, Nan, is involved in the recurved bristle-mediated mechanosensation. Different somatosensory bristles may use distinct molecular combinations in the mechanical-to-electric transduction to achieve proper sensitivity and potential redundancy.

\section{Central circuitry for the defensive behavior}

The wiring specificity of mechanosensory neurons ensures spatial precision essential for flies to detect the environment (Yorozu et al., 2009; Tuthill and Wilson, 2016). Our results revealed that brainless flies were able to achieve spatial precision in the kicking behavior, suggesting that the spatial representation is hardwired in the somatosensory-motor circuits of the VNC. Genetically hardwired neural circuits are essential for innate behaviors (Stockinger et al., 2005; Jefferis et al., 2007). Previous studies have shown that the fast escaping behavior is mainly mediated by the giant fiber system, which uses gap junctions to facilitate speedy response (Allen et al., 2006; von Reyn et al., 2014). The defensive behavior mediated by VNC alone provides a relatively simple system with which to further investigate how sensory neurons, interneurons, and motor neurons are connected and coordinated spatiotemporally to convert the sensory information into an accurate and fast behavioral output.

\section{References}

Akiyama T, Carstens MI, Ikoma A, Cevikbas F, Steinhoff M, Carstens E (2012) Mouse model of touch-evoked itch (alloknesis). J Invest Dermatol 132:1886-1891. CrossRef Medline

Albert JT, Göpfert MC (2015) Hearing in Drosophila. Curr Opin Neurobiol 34:79-85. CrossRef Medline

Allen MJ, Godenschwege TA, Tanouye MA, Phelan P (2006) Making an escape: development and function of the Drosophila giant fibre system. Semin Cell Dev Biol 17:31-41. CrossRef Medline

Benton R (2008) Chemical sensing in Drosophila. Curr Opin Neurobiol 18: 357-363. CrossRef Medline

Burrows M (1995) Motor patterns during kicking movements in the locust. J Comp Physiol A 176:289-305. Medline

Caldwell JC, Eberl DF (2002) Towards a molecular understanding of Drosophila hearing. J Neurobiol 53:172-189. CrossRef Medline

Chen S, Lee AY, Bowens NM, Huber R, Kravitz EA (2002) Fighting fruit 
flies: a model system for the study of aggression. Proc Natl Acad Sci U S A 99:5664-5668. CrossRef Medline

Corfas G, Dudai Y (1989) Habituation and dishabituation of a cleaning reflex in normal and mutant Drosophila. J Neurosci 9:56-62. Medline

Dunipace L, Meister S, McNealy C, Amrein H (2001) Spatially restricted expression of candidate taste receptors in the Drosophila gustatory system. Curr Biol 11:822-835. CrossRef Medline

Eberl DF, Hardy RW, Kernan MJ (2000) Genetically similar transduction mechanisms for touch and hearing in Drosophila. J Neurosci 20:59815988. Medline

Effertz T, Wiek R, Göpfert MC (2011) NompC TRP channel is essential for Drosophila sound receptor function. Curr Biol 21:592-597. CrossRef Medline

Fotowat H, Fayyazuddin A, Bellen HJ, Gabbiani F (2009) A novel neuronal pathway for visually guided escape in Drosophila melanogaster. J Neurophysiol 102:875-885. CrossRef Medline

Gaudry Q, Nagel KI, Wilson RI (2012) Smelling on the fly: sensory cues and strategies for olfactory navigation in Drosophila. Curr Opin Neurobiol 22:216-222. CrossRef Medline

Gong Z, Son W, Chung YD, Kim J, Shin DW, McClung CA, Lee Y, Lee HW, Chang DJ, Kaang BK, Cho H, Oh U, Hirsh J, Kernan MJ, Kim C (2004) Two interdependent TRPV channel subunits, inactive and Nanchung, mediate hearing in Drosophila. J Neurosci 24:9059-9066. CrossRef Medline

Gorczyca DA, Younger S, Meltzer S, Kim SE, Cheng L, Song W, Lee HY, Jan LY, Jan YN (2014) Identification of Ppk26, a DEG/ENaC channel functioning with Ppk1 in a mutually dependent manner to guide locomotion behavior in Drosophila. Cell Rep 9:1446-1458. CrossRef Medline

Goyal L, McCall K, Agapite J, Hartwieg E, Steller H (2000) Induction of apoptosis by Drosophila reaper, hid and grim through inhibition of IAP function. EMBO J 19:589-597. CrossRef Medline

Green D, Dong X (2016) The cell biology of acute itch. J Cell Biol 213:155161. CrossRef Medline

Guo Y, Wang Y, Wang Q, Wang Z (2014) The role of PPK26 in Drosophila larval mechanical nociception. Cell Rep 9:1183-1190. CrossRef Medline

Guo Y, Wang Y, Zhang W, Meltzer S, Zanini D, Yu Y, Li J, Cheng T, Guo Z, Wang Q, Jacobs JS, Sharma Y, Eberl DF, Göpfert MC, Jan LY, Jan YN, Wang Z (2016) Transmembrane channel-like (tmc) gene regulates Drosophila larval locomotion. Proc Natl Acad Sci U S A 113:7243-7248. CrossRef Medline

Hartenstein V, PosakonyJW (1989) Development of adult sensilla on the wing and notum of Drosophila melanogaster. Development 107:389-405. Medline

Hedwig B, Burrows M (1996) Presynaptic inhibition of sensory neurons during kicking movements in the locust. J Neurophysiol 75:1221-1232. Medline

Houck MA, Clark JB, Peterson KR, Kidwell MG (1991) Possible horizontal transfer of Drosophila genes by the mite Proctolaelaps regalis. Science 253: 1125-1128. CrossRef Medline

Jarman AP (2002) Studies of mechanosensation using the fly. Hum Mol Genet 11:1215-1218. CrossRef Medline

Jefferis GS, Potter CJ, Chan AM, Marin EC, Rohlfing T, Maurer CR Jr, Luo L (2007) Comprehensive maps of Drosophila higher olfactory centers: spatially segregated fruit and pheromone representation. Cell 128:11871203. CrossRef Medline

Jellema T, Heitler WJ (1999) Central and peripheral control of the trigger mechanism for kicking and jumping in the locust. J Comp Neurol 404: 212-220. Medline

Jenett A et al. (2012) A GAL4-driver line resource for Drosophila neurobiology. Cell Rep 2:991-1001. CrossRef Medline

Jones WD, Cayirlioglu P, Kadow IG, Vosshall LB (2007) Two chemosensory receptors together mediate carbon dioxide detection in Drosophila. Nature 445:86-90. CrossRef Medline

Kays I, Cvetkovska V, Chen BE (2014) Structural and functional analysis of single neurons to correlate synaptic connectivity with grooming behavior. Nat Protoc 9:1-10. CrossRef Medline

Kernan M, Cowan D, Zuker C (1994) Genetic dissection of mechanosensory transduction: mechanoreception-defective mutations of Drosophila. Neuron 12:1195-1206. CrossRef Medline

Kim J, Chung YD, Park DY, Choi S, Shin DW, Soh H, Lee HW, Son W, Yim J, Park CS, Kernan MJ, Kim C (2003) A TRPV family ion channel required for hearing in Drosophila. Nature 424:81-84. CrossRef Medline

Kim SE, Coste B, Chadha A, Cook B, Patapoutian A (2012) The role of Drosophila Piezo in mechanical nociception. Nature 483:209-212. CrossRef Medline
Lehnert BP, Baker AE, Gaudry Q, Chiang AS, Wilson RI (2013) Distinct roles of TRP channels in auditory transduction and amplification in Drosophila. Neuron 77:115-128. CrossRef Medline

Martin SJ, Highfield AC, Brettell L, Villalobos EM, Budge GE, Powell M, Nikaido S, Schroeder DC (2012) Global honey bee viral landscape altered by a parasitic mite. Science 336:1304-1306. CrossRef Medline

McNabb SL, Baker JD, Agapite J, Steller H, Riddiford LM, Truman JW (1997) Disruption of a behavioral sequence by targeted death of peptidergic neurons in Drosophila. Neuron 19:813-823. CrossRef Medline

Mendes CS, Bartos I, Akay T, Márka S, Mann RS (2013) Quantification of gait parameters in freely walking wild type and sensory deprived Drosophila melanogaster. eLife 2:e00231. CrossRef Medline

Muijres FT, Elzinga MJ, Melis JM, Dickinson MH (2014) Flies evade looming targets by executing rapid visually directed banked turns. Science 344:172-177. CrossRef Medline

Prieto-Godino LL, Benton R (2013) Neuroecology: a fly's bug detector. Curr Biol 23:R20-R22. CrossRef Medline

Raad H, Ferveur JF, Ledger N, Capovilla M, Robichon A (2016) Functional gustatory role of chemoreceptors in Drosophila wings. Cell Rep 15:1442-1454.

Ramdya P, Lichocki P, Cruchet S, Frisch L, Tse W, Floreano D, Benton R (2015) Mechanosensory interactions drive collective behaviour in Drosophila. Nature 519:233-236. CrossRef Medline

Sasaki K, Burrows M (2003) Proprioceptors monitoring forces in a locust hind leg during kicking form negative feedback loops with flexor tibiae motor neurons. J Exp Biol 206:759-769. CrossRef Medline

Seeds AM, Ravbar P, Chung P, Hampel S, Midgley FM Jr, Mensh BD, Simpson JH (2014) A suppression hierarchy among competing motor programs drives sequential grooming in Drosophila. eLife 3:e02951. CrossRef Medline

Seelig JD, Chiappe ME, Lott GK, Dutta A, Osborne JE, Reiser MB, Jayaraman V (2010) Two-photon calcium imaging from head-fixed Drosophila during optomotor walking behavior. Nat Methods 7:535-540. CrossRef Medline

Stockinger P, Kvitsiani D, Rotkopf S, Tirián L, Dickson BJ (2005) Neural circuitry that governs Drosophila male courtship behavior. Cell 121:795807. CrossRef Medline

Suh GS, Wong AM, Hergarden AC, Wang JW, Simon AF, Benzer S, Axel R, Anderson DJ (2004) A single population of olfactory sensory neurons mediates an innate avoidance behaviour in Drosophila. Nature 431:854859. CrossRef Medline

Tuthill JC, Wilson RI (2016) Parallel transformation of tactile signals in central circuits of Drosophila. Cell 164:1046-1059. CrossRef Medline

Valmalette JC, Raad H, Qiu N, Ohara S, Capovilla M, Robichon A (2015) Nano-architecture of gustatory chemosensory bristles and trachea in Drosophila wings. Sci Rep 5:14198. CrossRef Medline

Vandervorst P, Ghysen A (1980) Genetic control of sensory connections in Drosophila. Nature 286:65-67. CrossRef Medline

von Reyn CR, Breads P, Peek MY, Zheng GZ, Williamson WR, Yee AL, Leonardo A, Card GM (2014) A spike-timing mechanism for action selection. Nat Neurosci 17:962-970. CrossRef Medline

Walker RG, Willingham AT, Zuker CS (2000) A Drosophila mechanosensory transduction channel. Science 287:2229-2234. CrossRef Medline

Yamamoto D, Koganezawa M (2013) Genes and circuits of courtship behaviour in Drosophila males. Nat Rev Neurosci 14:681-692. CrossRef Medline

Yan Z, Zhang W, He Y, Gorczyca D, Xiang Y, Cheng LE, Meltzer S, Jan LY, Jan YN (2013) Drosophila NOMPC is a mechanotransduction channel subunit for gentle-touch sensation. Nature 493:221-225. CrossRef Medline

Yorozu S, Wong A, Fischer BJ, Dankert H, Kernan MJ, Kamikouchi A, Ito K, Anderson DJ (2009) Distinct sensory representations of wind and near-field sound in the Drosophila brain. Nature 458:201-205. CrossRef Medline

Yuan Q, Song Y, Yang CH, Jan LY, Jan YN (2014) Female contact modulates male aggression via a sexually dimorphic GABAergic circuit in Drosophila. Nat Neurosci 17:81-88. CrossRef Medline

Zhang W, Yan Z, Jan LY, Jan YN (2013) Sound response mediated by the TRP channels NOMPC, NANCHUNG, and INACTIVE in chordotonal organs of Drosophila larvae. Proc Natl Acad Sci U S A 110:13612-13617. CrossRef Medline

Zhang W, Cheng LE, Kittelmann M, Li J, Petkovic M, Cheng T, Jin P, Guo Z, Göpfert MC, Jan LY, Jan YN (2015) Ankyrin repeats convey force to gate the NOMPC mechanotransduction channel. Cell 162:1391-1403. CrossRef Medline 